\title{
Segmented phosphors: MEMS-based high quantum efficiency detectors for megavoltage $x$-ray imaging
}

\author{
Amit Sawant, ${ }^{\text {a) }}$ Larry E. Antonuk, Youcef El-Mohri, Yixin Li, Zhong Su, Yi Wang, \\ Jin Yamamoto, Qihua Zhao, and Hong Du \\ Department of Radiation Oncology, University of Michigan, Ann Arbor, Michigan 48103 \\ Jurgen Daniel and Robert Street \\ Palo Alto Research Center, Palo Alto, California
}

(Received 20 September 2004; revised 10 December 2004; accepted for publication 11 December 2004; published 3 February 2005)

Current electronic portal imaging devices (EPIDs) based on active matrix flat panel imager (AMFPI) technology use a metal plate+phosphor screen combination for x-ray conversion. As a result, these devices face a severe trade-off between $\mathrm{x}$-ray quantum efficiency $(\mathrm{QE})$ and spatial resolution, thus, significantly limiting their imaging performance. In this work, we present a novel detector design for indirect detection-based AMFPI EPIDs that aims to circumvent this trade-off. The detectors were developed using micro-electro-mechanical system (MEMS)-based fabrication techniques and consist of a grid of up to $\sim 2 \mathrm{~mm}$ tall, optically isolated cells of a photoresist material, SU-8. The cells are dimensionally matched to the pixels of the AMFPI array, and packed with a scintillating phosphor. In this paper, various design considerations for such detectors are examined. An empirical evaluation of three small-area $\left(\sim 7 \times 7 \mathrm{~cm}^{2}\right)$ prototype detectors is performed in order to study the effects of two design parameters - cell height and phosphor packing density, both of which are important determinants of the imaging performance. Measurements of the x-ray sensitivity, modulation transfer function (MTF) and noise power spectrum (NPS) were performed under radiotherapy conditions $(6 \mathrm{MV})$, and the detective quantum efficiency (DQE) was determined for each prototype SU-8 detector. In addition, theoretical calculations using Monte Carlo simulations were performed to determine the QE of each detector, as well as the inherent spatial resolution due to the spread of absorbed energy. The results of the present studies were compared with corresponding measurements published in an earlier study using a Lanex Fast-B phosphor screen coupled to an indirect detection array of the same design. The SU-8 detectors exhibit up to 3 times higher QE, while achieving spatial resolution comparable or superior to Lanex Fast-B. However, the DQE performance of these early prototypes is significantly lower than expected due to high levels of optical Swank noise. Consequently, the SU-8 detectors presently exhibit DQE values comparable to Lanex Fast-B at zero spatial frequency and significantly lower than Fast-B at higher frequencies. Finally, strategies for reducing Swank noise are discussed and theoretical calculations, based on the cascaded systems model, are presented in order to estimate the performance improvement that can be achieved through such noise reduction. () 2005 American Association of Physicists in Medicine. [DOI: 10.1118/1.1854774]

Key words: segmented detectors, quantum efficiency, portal imaging, active matrix flat panel imager, DQE

\section{INTRODUCTION}

Precise patient positioning and accurate dose delivery are critical aspects of external beam radiotherapy. Presently, the most common technique for achieving these objectives is portal imaging, which involves using the megavoltage $\mathrm{x}$-ray beam to acquire projection images (often through the treatment window or portal) before and, optionally, during dose delivery. Following over 15 years of extensive research and development, indirect detection active-matrix flat-panel imager (AMFPI)-based electronic portal imaging devices (EPIDs) have become the current gold standard in portal imaging, providing imaging performance superior to that of conventional portal film as well as earlier, commercial EPID technologies. ${ }^{1,2}$ Currently, all commercially-available
AMFPI-based portal imaging systems are based on indirect detection, i.e., using a scintillator coupled to a photodiode array. ${ }^{3}$ While there have been several studies examining the feasibility of direct detection AMFPIs based on amorphous selenium ( $a$-Se) and other converters for portal imaging, these approaches are still under investigation. ${ }^{4-8}$

The success of AMFPIs in portal imaging can be largely attributed to the fact that they are x-ray quantum limited, i.e., the imaging performance of these systems is ultimately limited by the statistical noise of the x-ray quanta detected by the converter. ${ }^{9,10}$ Nonetheless, there exists considerable scope for improving the performance of AMFPI-based portal imagers due to the fact that these devices utilize only $\sim 1 \%-2 \%$ of the incident radiation to form an image. ${ }^{3}$ This low $\mathrm{x}$-ray quantum efficiency $(\mathrm{QE})$ is due to the fact that 
current AMFPI systems use a metal plate+phosphor screen combination as the $\mathrm{x}$-ray converter. ${ }^{3}$ The use of a phosphor screen introduces a severe trade-off between $\mathrm{x}$-ray detection efficiency (which increases with increasing screen thickness) and spatial resolution (which decreases with increasing screen thickness). ${ }^{11-13}$ Consequently, the imaging performance of AMFPI EPIDs, as quantified by the frequencydependent detective quantum efficiency $[\mathrm{DQE}(\mathrm{f})]$, is relatively modest when compared to AMFPIs designed for diagnostic energies. [For example, the DQE(0) for megavoltage AMFPI systems is $\sim 1 \%$, $10,13,14$ while that for diagnostic AMFPIs ranges from $50 \%$ to $75 \%{ }^{15-18}$ ]

A variety of strategies to significantly improve EPID performance by using high QE detectors have been examined. These include modifications to TV-camera-based systems involving the replacement of the conventional phosphor screen with a thick, grooved phosphor screen, ${ }^{19}$ with a segmented 2D array of $\mathrm{CsI}(\mathrm{Tl})$ elements ${ }^{20,21}$ or, with a large-area $\mathrm{CsI}(\mathrm{Tl})$ crystal in a novel geometrical arrangement; ${ }^{22}$ and using high-pressure gas chamber-based detectors in scanning ${ }^{23,24}$ and area detection ${ }^{25-27}$ EPID systems. Studies involving segmented scintillator crystals in a 1D configuration coupled to a photodiode array ${ }^{28}$ and in a $2 \mathrm{D}$ configuration coupled to an indirect detection active matrix array ${ }^{29}$ have also been reported for megavoltage computed tomography. In addition, high QE detectors for direct detection-based AMFPI EPIDs are currently under investigation. ${ }^{30}$ (Comprehensive reviews on these and other performance enhancement strategies, as well as early EPID designs, may be found elsewhere. ${ }^{3,31,32}$ )

In this paper, we present an empirical study of a novel high QE detector design based on micro-electro-mechanical system (MEMS) fabrication techniques, for indirect detection-based AMFPI EPIDs. In principle, such a design enables the use of a thick layer of scintillating phosphor without the accompanying trade-off in spatial resolution. In the following sections, the general concept and various design considerations are described, followed by measurements performed on early prototype detectors to determine $\mathrm{x}$-ray sensitivity, modulation transfer function (MTF), noise power spectra (NPS), and DQE. Finally, we examine strategies to further enhance the imaging performance of these detectors and present theoretical calculations in order to predict the upper limits of performance that can be achieved.

\section{TECHNICAL BACKGROUND}

\section{A. High QE detectors using segmented phosphors}

In order to circumvent the trade-off between $\mathrm{QE}$ and spatial resolution for phosphor-based MV detectors, we are investigating a detector design that incorporates a two dimensional matrix of cells which are dimensionally matched and registered to the pixels of the underlying indirect detection array (as shown in Fig. 1). The inner walls of the cells are designed to be opaque, to achieve optical isolation between adjacent cells, and reflective, in order to increase the number of light photons impinging on the underlying pixels. Under ideal conditions, when such a matrix is packed with a scin-

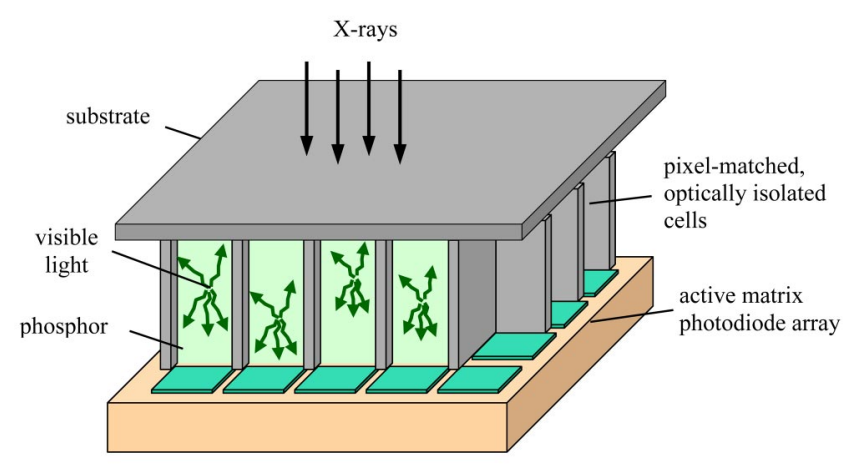

FIG. 1. Schematic illustration of the segmented phosphor concept. The detector consists of a 2D matrix of optically isolated cells that are dimensionally matched to the pixels of the underlying indirect detection flat panel array. The matrix is filled with a scintillating phosphor and coupled to the array such that the detector cells are accurately registered to the array pixels.

tillating phosphor, the light generated within a cell due to $\mathrm{x}$-ray interactions is confined to that cell and can only be detected by the underlying pixel. In principle, such an arrangement enables the use of a thick layer of phosphor without the accompanying loss of spatial resolution due to the spread of optical photons. The implementation of this concept requires careful optimization with respect to several detector parameters such as cell height, the optical properties of the cell walls, the phosphor material, the packing density of the phosphor grains, etc. In the design approach adopted for this work, these parameters have been examined in the context of optimizing the DQE(f) of the imaging system.

\section{B. Design considerations: The DQE approach to detector design}

The spatial frequency-dependent detective quantum efficiency DQE(f) is a widely accepted metric of $x$-ray imaging performance. ${ }^{33} \mathrm{DQE}$ is a measure of the efficiency of information transfer from the input to the output of an imaging system, and is related to spatial resolution (i.e., the MTF), the gain and the noise transfer properties (i.e., the NPS) of a system as follows: ${ }^{34}$

$$
\mathrm{DQE}=\frac{q_{0} \times \operatorname{gain}^{2} \times \mathrm{MTF}^{2}}{\mathrm{NPS}},
$$

where $q_{0}$ is the total number of incident quanta per unit area. The DQE can also be expressed as the ratio of the squared output signal to noise ratio (SNR) to the squared input SNR. An x-ray imaging system can be considered to be composed of a cascade of linearly coupled stages. ${ }^{35,36}$ In such a view, the input signal and noise are transferred through each stage via a combination of amplifying and/or scattering processes, with the output of each stage serving as input to the subsequent stage. From the above description it is clear that, in order to maximize the squared SNR transfer efficiency (i.e., the DQE) of the entire imaging system, it is necessary to optimize SNR transfer in the detector. In the context of this paper, we shall refer to the squared SNR transfer efficiency of the detector as the "detector DQE" and that of the entire 
system as the "system DQE." From Eq. (1) it is clear that, in order to achieve good DQE performance, a detector should exhibit high gain and spatial resolution, while maintaining low noise levels. In the remainder of this section, we examine the various design considerations in the context of these requirements.

\section{Optimizing detector gain}

The gain of a scintillator-based detector is the product of three components - the X-ray quantum efficiency $(\mathrm{QE})$ of the detector, the conversion gain (i.e., the average number of light quanta produced per x-ray interaction), and the escape efficiency (i.e., the average probability that a light photon exits from the array side of the scintillator). For x-ray quantum limited systems (such as AMFPI EPIDs), the gain component that serves to improve DQE is the quantum efficiency of the detector. 9,36 The QE can be improved through a combination of any or all of the following strategies: (a) using a high-electron-density phosphor material, (b) increasing the thickness of the phosphor layer by fabricating taller cells, (c) increasing the "fill factor" of the cell by making the cell walls as thin as mechanically feasible, and (d) increasing the packing density of the phosphor grains in the cells. The implementation of strategy (d) has to be based on a trade-off between quantum efficiency, which increases with higher packing density, and the escape efficiency of the optical photons, which decreases with higher packing density. In a more densely packed phosphor, optical photons undergo more scattering and absorption events, leading to two effects. First, the overall light output of the converter is reduced. However, reduced light output in itself does not constitute a significant problem as long as the system remains x-ray quantum limited. More importantly, high levels of scattering and absorption give rise to a strongly depth-dependent optical gain. As explained in Sec. B 3, a depth-dependent gain is highly undesirable as it serves to increase the noise and thereby, reduce the DQE.

\section{Optimizing spatial resolution (MTF)}

The structured scintillator design constrains the light generated due to $\mathrm{x}$-ray interactions within a cell, thus limiting the loss in spatial resolution due to the spread of the optical photons. As the thickness of the phosphor layer increases up to a few centimeters, other resolution-loss mechanisms, such as the spreading of the absorbed energy and parallax effects due to the obliquely incident off-axis $\mathrm{x}$ rays, become significant. However, for the detector thicknesses discussed in the present work (up to $\sim 2 \mathrm{~mm}$ ), these effects contribute minimally and the overall loss in spatial resolution is dominated by optical spreading. In order to limit the spread of optical photons, it is essential to maintain good optical isolation (i.e., minimize optical cross-talk) between adjacent cells. It is also important to achieve good optical coupling between the detector and the array, as well as accurate registration between the scintillator cells and the underlying array pixels.

\section{Optimizing noise properties (NPS)}

A phosphor-based detector exhibits variation in the number of optical quanta emitted per interacting x-ray. The noise originating from this variation is termed as Swank noise, and is quantified by the Swank factor (which ranges between 0 and 1). ${ }^{37}$ Swank noise degrades DQE performance across all spatial frequencies. The major factors that contribute to Swank noise are (a) the x-ray energy distribution (XED), (b) the absorbed energy distribution (AED), and (c) the optical pulse distribution (OPD). For screen thicknesses comparable to those used in conventional radiotherapy AMFPIs [ $\sim 360 \mu \mathrm{m}$ of $\mathrm{Gd}_{2} \mathrm{O}_{2} \mathrm{~S}: \mathrm{Tb}$ at $50 \%$ density (Refs. 3 and 10 )], the Swank noise is dominated by the XED and the AED. ${ }^{10}$ However, as the thickness of the phosphor layer and the packing density of the phosphor grains increase, the contribution of the OPD becomes more significant. For thicker screens, there is a large variation in the amount of scatter and absorption (and therefore, the escape efficiency) of light quanta generated at various depths within the phosphor. This variation increases the Swank noise due to widening of the OPD. Thus, in order to reduce Swank noise, it is necessary to optimize the packing density as well as the optical properties of the phosphor so as to make the number of light quanta escaping the detector minimally dependent on the depth at which they are generated. ${ }^{38}$

Another effect caused by the depth-dependent nature of the optical transport is the Lubberts effect. ${ }^{39}$ This effect pertains to the variation in the shape of the point spread function (PSF) observed at the output, for optical quanta generated at various depths within the phosphor. According to Lubberts, a thick phosphor detector can be considered to be composed of multiple layers of phosphor which are sufficiently thin such that, within a layer, there is no significant depth-dependent variation of the optical PSF. In such a view, the optical component of the MTF of the entire detector is proportional to the (weighted) sum of the optical MTFs of the individual layers

$$
T_{\mathrm{opt}}(u) \propto \frac{\sum_{i=1}^{N} T_{i}(u)}{N},
$$

where $T_{\text {opt }}(u)$ is the optical MTF of the detector as a function of spatial frequency, $T_{i}(u)$ is the optical MTF of the $i$ th layer, and $N$ is the total number of layers. In contrast, the optical component of the NPS of the detector, $S_{\text {opt }}(u)$, is proportional to the sum of the squares of the individual MTFs:

$$
S_{\text {opt }}(u) \propto \frac{\sum_{i=1}^{N} T_{i}^{2}(u)}{N} .
$$

Equations (2) and (3) shown above are simplified forms of Eqs. (8) and (7), respectively, in Nishikawa et al. ${ }^{40}$

As a result of this dissimilar integration across the layers of the detector, $T_{\mathrm{opt}}^{2}(u)$ falls off faster with respect to spatial frequency than $S_{\text {opt }}(u)$. As the detector thickness increases, the inequality between the fall-off of MTF and the NPS becomes more pronounced. Consequently, the DQE, which is directly proportional to the square of the MTF and inversely proportional to the NPS [as indicated in Eq. (1)], exhibits a 


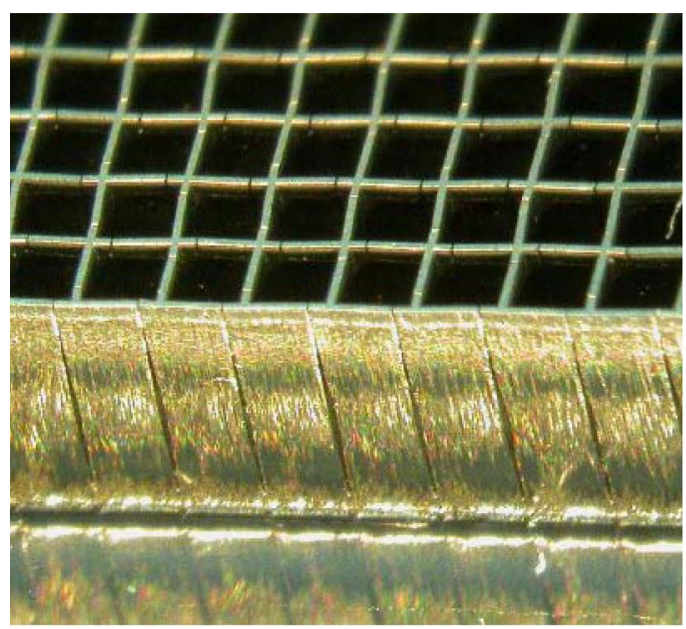

(a)

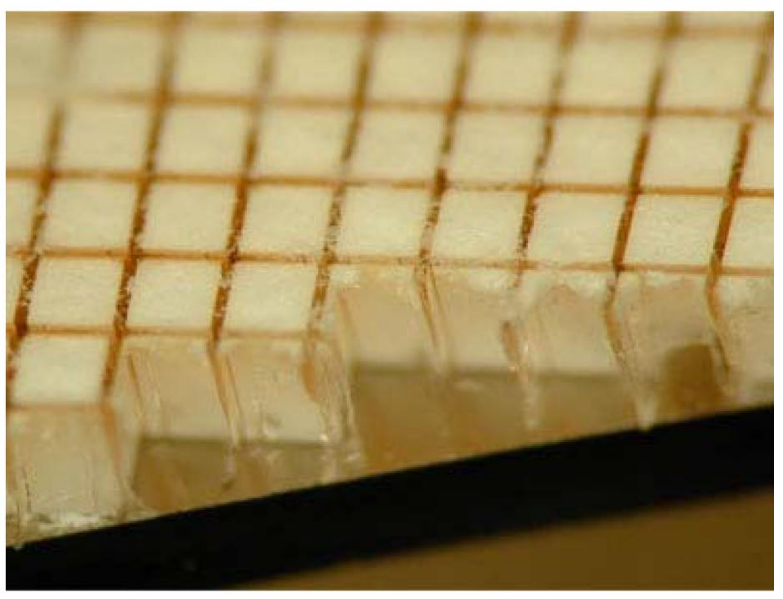

(b)

FIG. 2. Microphotographs of small portions of two SU-8 cell matrices. The cells have a pitch of $508 \mu \mathrm{m}$, which is equal to the pixel pitch of the active matrix array used in this work. (a) Cells prior to phosphor deposition. The cell walls are sputtered with Al in order to make them opaque and reflective. Note the small gaps $(\sim 10 \mu \mathrm{m})$ in the cell walls. These gaps are incorporated in order to facilitate uniform phosphor deposition, and are offset such that there is no straight optical path between more than two adjacent cells. (b) Cells after phosphor deposition, from a different matrix. In this case, the walls have not been metallized in order to better illustrate the phosphor. A portion of this matrix has been diced in order to examine the uniformity of the phosphor deposition.

faster drop at higher spatial frequencies. ${ }^{39,40}$ In order to minimize the Lubberts effect, it is essential that the optical MTF be practically independent of the depth of light generation. ${ }^{40}$ In the context of a segmented scintillator, this goal can be achieved by ensuring good optical isolation between cells, making the cell walls highly reflective and carefully optimizing the optical properties of the phosphor so as to minimize scattering and absorption (thereby, maximizing the mean free path) of the generated light photons.

\section{METHODS AND MATERIALS}

\section{A. Fabrication of prototype detectors}

In order to examine the segmented phosphor concept, prototype detectors were fabricated using photolithographic techniques. A recently developed photopolymer SU-8, ${ }^{41}$ which is well-suited to large-area photolithographic processing methods, was used to create high-aspect-ratio cell matrices on a $10 \mathrm{~cm}$ diameter glass substrate. ${ }^{42-44}$ The matrices (shown in Fig. 2) were composed of $\sim 135 \times 135$ cells that were dimensionally matched to the pixel pitch $(508 \mu \mathrm{m})$ of an indirect detection flat panel array previously developed for radiotherapy imaging. ${ }^{1,10}$ The inner walls of the cells and the inner surface of the glass substrate were made opaque and reflective by sputtering a thin layer of Al. (The thickness of the Al layer was on the order of a few microns.) The cell walls were designed to be $\sim 50 \mu \mathrm{m}$ thick in order to achieve a balance between mechanical strength and fill-factor, as discussed in Sec. II B 1.

In the present study, we examined two important parameters of detector design that are strong determinants of the imaging performance of a segmented detector-the cell height and the packing density of the phosphor grains. The phosphor used in the present study was $\mathrm{Gd}_{2} \mathrm{O}_{2} \mathrm{~S}: \mathrm{Tb}$ (GOS) in powder form, with grain sizes of $\sim 3$ to $7 \mu \mathrm{m}$. Three dif- ferent detector configurations were studied. Two of these were high-density (HD) detectors, filled with $\sim 75 \%$ GOS by volume, with cell heights (i.e., detector thicknesses) equal to $850 \mu \mathrm{m}$ (HD-1) and $1900 \mu \mathrm{m}$ (HD-2). The third detector was a low-density (LD) configuration which consisted of $1900 \mu \mathrm{m}$ tall cells filled with $\sim 35 \%$ GOS. (In the context of this paper, the term "SU-8 detectors" will generally be used to indicate detectors based on the photopolymer SU-8, while "HD-1," "HD-2" and "LD" will be used to indicate the specific detector configurations described above.) A novel technique (described below) was developed to achieve reasonably uniform phosphor deposition in these high-aspect-ratio cell matrices [see Fig. 2(b)], with slightly different versions of the technique used in the case of the HD and the LD detectors. In all cases, the SU-8 cell matrix was initially filled with a paraffin-based solvent (Isopar, ExxonMobil Corp.) which has low viscosity and good wetting properties, in order to minimize the formation of air pockets during the phosphor deposition.

In the case of the HD detectors, a slurry was prepared (in a separate container) by dispersing the GOS powder in the solvent, along with a small amount of dispersant (Solsperse, Avecia, Inc.). The dispersant was used in order to prevent particle agglomeration so as to facilitate uniform deposition of the slurry into the cell matrix. The slurry was degassed under vacuum and carefully poured into the SU-8 matrix. After filling, the SU-8 matrix was placed in the vacuum chamber in order to purge air bubbles (since large, non uniformly distributed air pockets would increase Swank noise due to effects described in Sec. II B 3 and subsequently placed in an oven at a temperature of $\sim 65^{\circ} \mathrm{C}$ in order to evaporate the solvent. The total volume of all the cells in each detector was calculated (using the cell height and the number of cells in the detector), and the weight of the detector before and after filling was measured in order to estimate 
the packing density of the GOS phosphor, which was determined to be $\sim 75 \%$. Finally, a thin layer of UV-curable optical glue was applied on the open surface of the cell matrix and was allowed to partially penetrate the phosphor (through capillary action) in order to bind the phosphor grains together. The glue was then cured using a long-wave $(365 \mathrm{~nm})$ UV light source.

The UV-curable glue (OP-401, Dymax Corp.) was chosen for its relatively low viscosity $(70 \mathrm{cP})$, which facilitates penetration into the phosphor, as well as high optical clarity and high index of refraction $(\sim 1.5)$ in the cured state, both of which serve to improve optical transport within the cells. In addition, the optical transport is also likely to be affected by the depth and the uniformity of penetration of the glue. For samples fabricated using the above phosphor deposition technique, our measurements indicate that the glue penetrates $\sim 300 \pm 25 \mu \mathrm{m}$ from the open surface of the detector.

For the LD detector, the GOS powder was directly dispersed in the optical glue in order to achieve the desired packing density $(\sim 35 \%)$ and the resulting slurry was deposited into the SU-8 matrix, degassed and cured, in a manner similar to that described for the HD detectors. After phosphor deposition, each detector was lightly polished in order to make the surface uniform so as to facilitate good optical coupling between the detector and the array.

\section{B. Measurements}

All measurements were performed with a $6 \mathrm{MV}$ photon beam, on a Varian 21-EX linear accelerator (linac), using a $512 \times 512$ pixel, 508 micron pitch $(83 \%$ optical fill factor) indirect detection active matrix array developed for radiotherapy applications. ${ }^{1,10}$ The array was connected to a fully customized electronic acquisition system, ${ }^{45}$ which included custom-designed preamplifier-multiplexers ${ }^{46}$ previously developed for portal imager development using such arrays. In each case, the SU-8 detector was placed on the array such that the open surface of the cell matrix was in contact with the array pixels. In addition, an overlying $1 \mathrm{~mm}$ thick $\mathrm{Cu}$ plate was coupled to the SU-8 detector in order to provide build-up as well as to absorb scattered, low-energy electrons and photons. ${ }^{3}$ Each detector was aligned under an optical microscope to the underlying flat-panel array using fiducial cross-hairs (as shown in Fig. 3) so as to achieve registration of the detector cells to the array pixels. The registration was verified under the x-ray beam using isolated rows of phosphor filled cells located on the four sides of the detector, as shown in Fig. 3. Signal and noise measurements were performed as a function of the irradiation time in terms of monitor units (MU) of the linac. [For this linac, $1 \mathrm{MU}$ corresponds to a dose of $0.8 \mathrm{cGy}$ deposited in water at a sourceto-detector (SDD) equal to $100 \mathrm{~cm}$, with $10 \mathrm{~cm}$ overlying water, for a field size of $10 \times 10 \mathrm{~cm}^{2}$ at the isocenter (i.e., $100 \mathrm{~cm} \mathrm{SDD).]} \mathrm{For} \mathrm{the} \mathrm{signal} \mathrm{and} \mathrm{noise} \mathrm{measurements,} \mathrm{the}$ AMFPI was operated in fluoroscopic mode and the radiation was delivered at a dose rate of $100 \mathrm{MU} / \mathrm{min}$. The MTF data was acquired in radiographic mode, with the linac delivering radiation at $600 \mathrm{MU} / \mathrm{min}$ in order to accommodate the rela-

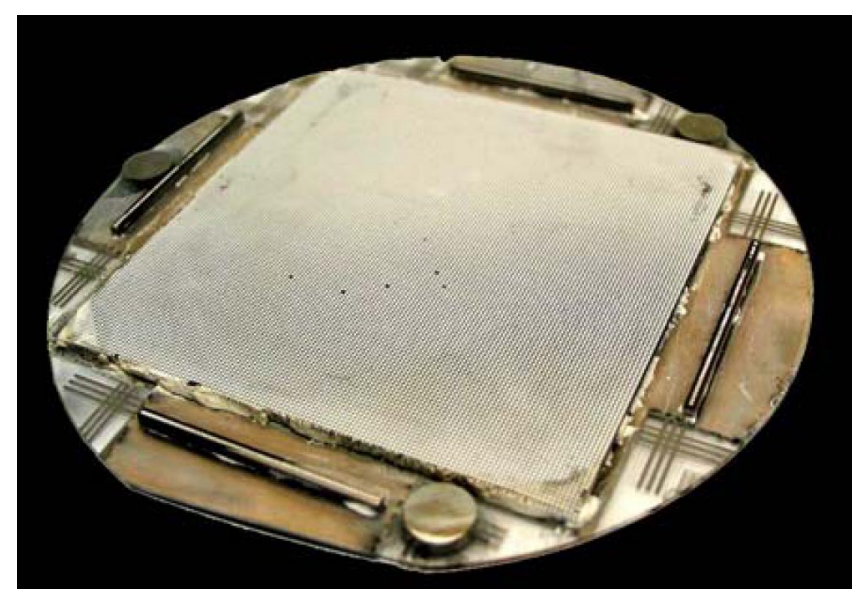

FIG. 3. Photograph of the HD-2 detector after phosphor deposition and polishing. In order to incorporate it into the imager, the detector is inverted and coupled to the active matrix array. The horizontal and vertical cross-hairs at the corners serve to register the detector cells to the array pixels. The alignment is verified under the x-ray beam with respect to the isolated rows of cells (also filled with phosphor) present on each side of the matrix. The dark spots in the center of the matrix correspond to incompletely-filled cells.

tively large amounts of irradiation required for the MTF measurements in a reasonable frame time. (Details of the fluoroscopic and radiographic operation of this array may be found in El-Mohri et al. ${ }^{10}$ )

\section{Sensitivity}

Sensitivity was determined in terms of the signal response of the system per monitor unit (MU). The imager (i.e., the detector+flat panel array) was placed at a source-to-detector distance (SDD) equal to $130 \mathrm{~cm}$ with a field size of 10 $\times 10 \mathrm{~cm}^{2}$ at the isocenter. Image frames were acquired by synchronizing the data acquisition with the delivery of a predetermined number of beam pulses $(18,36$, and 72$)$ corresponding to $0.5,1$, and $2 \mathrm{MU}$, respectively. (The number of monitor units per pulse, $\approx 0.028$, was determined by counting the number of pulses from the "Target I" output from the linac for a programmed MU setting.) For each data sequence, a total of 100 dark and flood data frames (i.e., frames in the absence and presence of radiation, respectively) were acquired. For each data set, the average dark frame was subtracted from the average flood frame to yield the signal. The signal values were converted from ADC units to electrons using a measured calibration factor $(1 \mathrm{ADC} \approx 7480 \mathrm{e})$ for the preamplifiers of the data acquisition system. The slope of the signal response plotted as a function of the irradiation time yielded the sensitivity in units of e/MU.

\section{Modulation transfer function (MTF)}

MTF for the three prototype detectors was measured using the angled slit technique, ${ }^{47}$ adapted for imaging at megavoltage $\mathrm{x}$-ray energies. ${ }^{9,10,14}$ A long, narrow slit of dimensions $20 \times 0.01 \mathrm{~cm}^{2}$ was formed using two $20 \times 10 \times 5 \mathrm{~cm}^{3}$ precision-ground steel blocks separated by $0.01 \mathrm{~cm}$ shims. The slit was centered with respect to the radiation source in order to maximize the signal, and positioned such that the 
exit surface of the slit was almost in contact with the detector, which was placed at a SDD of $86 \mathrm{~cm}$. The field size was adjusted to $6 \times 6 \mathrm{~cm}^{2}$ at the exit surface of the slit. Image frames were acquired by operating the imager in the radiographic mode with relatively long irradiations $(\sim 18 \mathrm{MU})$ in order to obtain sufficient signal through the slit relative to the background. For each prototype detector, 10 images were acquired. The steel blocks attenuated $\sim 99.3 \%$ of the beam, and the effect of the remaining radiation penetrating through the blocks was determined by displacing the slit $\sim 0.6 \mathrm{~cm}$ away from the center and acquiring "radiation profile" images. Gain and offset corrections were applied to all image data sets and the radiation profile images were subtracted from the slit images. For each prototype detector, the final, corrected images were averaged and used to estimate the line spread function (LSF). The baseline of the LSF was processed by selectively using a $3 \times 3$ median filter around portions that exhibited sharp spikes (due to nonfunctional pixels) and the absolute value of the Fourier transform of the LSF yielded the one-dimensional MTF.

\section{Noise power spectrum (NPS)}

Noise power measurements were performed using the same setup used for the sensitivity measurements. Dark and flood image frames were acquired fluoroscopically at 1 and 2 MU (i.e., at 36 and 72 linac pulses per data frame, respectively), with up to 800 data frames per acquisition sequence. Each flood frame was corrected using gain and offset constants obtained from the dark and flood data. ${ }^{48}$ A $3 \times 3$ median filter was applied in order to correct for defective array pixels and incompletely filled detector cells, with a threshold set in order to ensure that less than $0.2 \%$ of the total number of pixels were filtered per frame. It has been found that, for indirect detection AMFPIs, the use of such a filter to remove up to $0.5 \%$ of the pixels has minimal impact on the noise power characteristics. ${ }^{49}$ The pixel ADC values were converted into electrons as described in the previous section. One-dimensional noise power spectra were determined from these images using the synthesized slit technique, which is described in detail elsewhere ${ }^{10,49}$ and briefly summarized as follows. 800 independent, nonoverlapping blocks (i.e., slits), each with $40 \times 100$ pixels, were selected. Each slit was summed along the narrow direction to form a 100-point realization. Low frequency background trends were subtracted and a Hanning window was applied to each realization. A 1D Fourier transform was applied to each of the 800 realizations and the resulting power spectra, were appropriately normalized and averaged to yield the fluoroscopic 1D NPS. Subsequently, the NPS data were corrected for the effect of first frame lag (determined to be $\sim 10 \%$ ) in order to compensate for the noise-reduction effect caused by frame-to-frame charge carryover (i.e., lag). Details of the correction technique may be found elsewhere. ${ }^{50,51}$

\section{Detective quantum efficiency (DQE)}

The frequency-dependent DQE was determined for each detector configuration using the measured NPS (with lag correction, as described above) and MTF. The DQE was calculated using the relation

$$
\operatorname{DQE}(u)=\frac{A^{2} T_{\mathrm{sys}}^{2}(u)}{q_{0} S_{\mathrm{sys}}(u)},
$$

where $u$ represents the independent spatial frequency variable along one axis, $A$ is the average signal per pixel (obtained from the flood frames used to calculate the NPS), $q_{0}$ is the incident X-ray fluence, while $T_{\text {sys }}(u)$ and $S_{\text {sys }}(u)$ represent the one-dimensional MTF and NPS of the system, respectively.

In addition, the degradation of DQE at non-zero spatial frequencies due to Lubberts effect (described in Sec. II B 3 was determined by calculating a factor, $R(u)$, previously defined by Nishikawa et ll. $^{40}$ as

$$
R(u)=\frac{T_{\text {sys }}^{2}(u)}{S_{\text {profile }}(u)},
$$

where $S_{\text {profile }}(u)$ gives the shape of the system NPS:

$$
S_{\text {profile }}(u)=\frac{S_{\text {sys }}(u)}{S_{\text {sys }}(0)} .
$$

From Eqs. (5) and (6) it is clear that, for an ideal system that does not exhibit Lubberts effect, $R(u)$ will be unity at all spatial frequencies.

\section{Monte Carlo based theoretical calculations}

The x-ray quantum efficiency of the three detector configurations was estimated by simulating $\mathrm{x}$-ray photon and electron transport using the EGS4 Monte Carlo codes ${ }^{52}$ and the RZ_PHS user code, ${ }^{53}$ which scores the distribution of the energy absorbed within a detector (i.e., the AED) for an incident $\mathrm{x}$-ray energy spectrum. A $6 \mathrm{MV}$ photon spectrum $^{54}$ corresponding to a Varian linac was used in all simulations. One million photon histories were used for each simulation, which resulted in statistical uncertainties of less than $2 \%$. The modeled geometry consisted of a pencil beam perpendicularly incident on the center of a cylindrical slab of $40 \mathrm{~cm}$ diameter. The slab consisted of a $1 \mathrm{~mm} \mathrm{Cu}$ layer coupled to an underlying layer of phosphor (GOS). The thickness of the GOS layer was chosen to be equal to the measured values, 850, 1900, and $1900 \mu \mathrm{m}$, for the HD-1, HD-2, and the LD detector, respectively. The density of the phosphor material was determined by comparing the packing density of the phosphor grains with the bulk density of GOS $\left(7.34 \mathrm{~g} / \mathrm{cm}^{3}\right)$.

Since the phosphor was modeled as a continuous layer (i.e., x-ray interactions in the cell walls were not considered, given the relatively low density, $\sim 1 \mathrm{~g} / \mathrm{cm}^{3}$, of SU-8), the measured packing densities $(75 \%$ and $35 \%$ for the HD and LD detectors, respectively) were scaled by the geometric fill factor $(\sim 0.81)$ of the SU-8 cells, and the corrected values were used to calculate the equivalent phosphor density for the HD $\left(\sim 4.40 \mathrm{~g} / \mathrm{cm}^{3}\right)$ and the LD $\left(2.05 \mathrm{~g} / \mathrm{cm}^{3}\right)$ detectors. 
The x-ray quantum efficiency of the detector was calculated from the ratio of the number of photons that deposit energy in the phosphor layer to the number of incident photons, as determined from the Monte Carlo simulations.

In addition, for each configuration, Monte Carlo simulations were also performed in order to estimate the spread of the absorbed energy within the detector. The EGSnrc Monte Carlo codes ${ }^{55}$ and the DOSXYZnrc user code ${ }^{56}$ were used to estimate the LSF, and thereby the MTF, of the absorbed energy, referred to hereafter as the "radiation MTF." The aforementioned $6 \mathrm{MV}$ photon spectrum was used (with 200 million histories) to form a narrow, parallel slit beam of dimensions $40 \times 0.004 \mathrm{~mm}^{2}$. The beam was made incident on a $40 \times 40 \mathrm{~mm}^{2}$ slab consisting of a $1 \mathrm{~mm}$ thick $\mathrm{Cu}$ plate overlying the detector. The LSF was obtained by scoring the absorbed energy along a $1 \times 40 \mathrm{~mm}^{2}$ strip in the center of the detector, perpendicular to the narrow slit formed on the detector plane by the incident beam. In order to ensure sufficient sampling of the LSF, the strip was divided into 8000 voxels, each $1 \times 0.005 \mathrm{~mm}^{2}$. The LSF, thus obtained, was used to calculate the radiation MTF.

\section{RESULTS}

The results obtained from the measurements performed on the SU-8 detectors are reported below. In order to establish a context for these results, they are compared with previously reported data obtained at $6 \mathrm{MV}$, using a Lanex Fast-B phosphor screen (typical of those used in most clinical AMFPI EPID systems ${ }^{3}$ ) coupled to an array of similar design. ${ }^{10}$

\section{A. Sensitivity}

Figure 4(a) shows the signal response for various detectors, in units of electrons, as a function of irradiation time in terms of monitor units. The lines represent a linear fit to the data. It can be seen that the signal response of the three SU-8 detector configurations exhibits good linearity. Among the three configurations, HD-1 shows the highest signal levels, followed by HD-2 and finally, LD. In addition, the sensitivity profile of a central region of the HD-2 detector is shown in Fig. 4(b). The relatively small variations in the sensitivity profile indicate that the phosphor deposition is fairly uniform over most of the cell matrix. It should be noted that these variations in the sensitivity profile are almost entirely due to non-uniformities in the SU-8 detector, as indirect detectionbased active matrix arrays typically exhibit very uniform optical response. ${ }^{57}$ This point is illustrated in Fig. 4(c), which shows a sensitivity profile obtained from the same region of the array when coupled to a commercial phosphor screen (Lanex Fast-B).

Table I summarizes various detector parameters obtained from measurements and Monte Carlo simulations. From the data presented in Fig. 4 and the values summarized in Table I, it can be seen that the x-ray sensitivity of the segmented phosphors is affected by a combination of the QE and the optical transport characteristics of the phosphor. For instance, while the HD-1 and LD configurations have approxi-

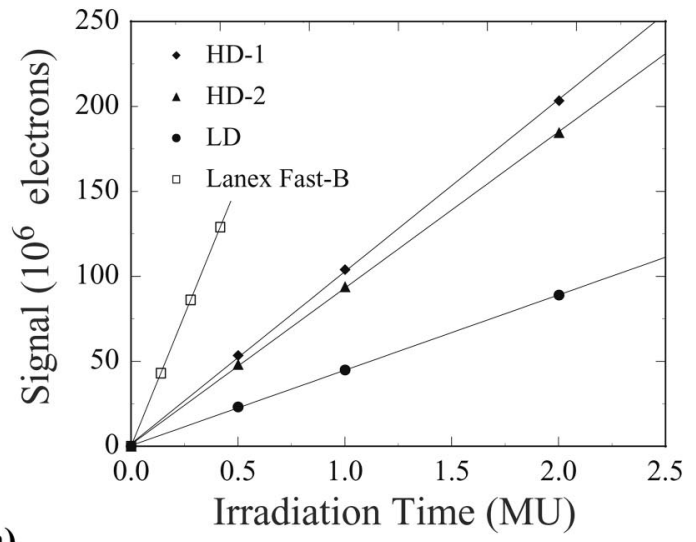

a)

b)

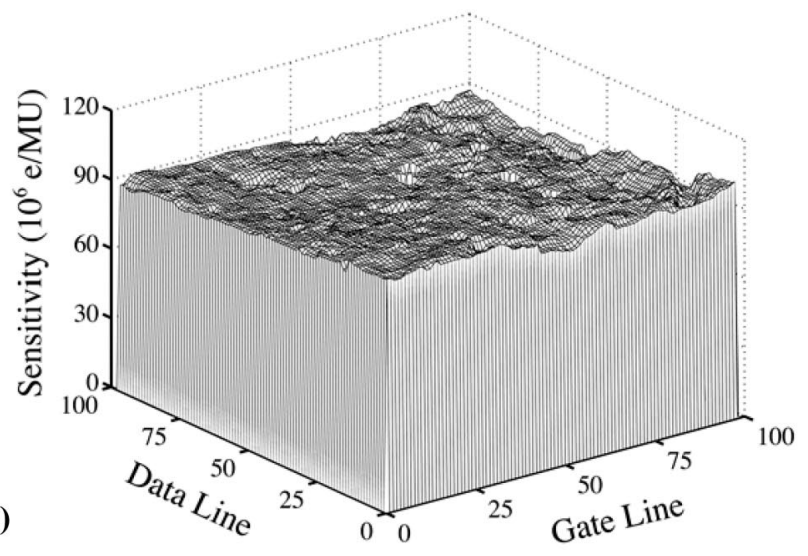

c)

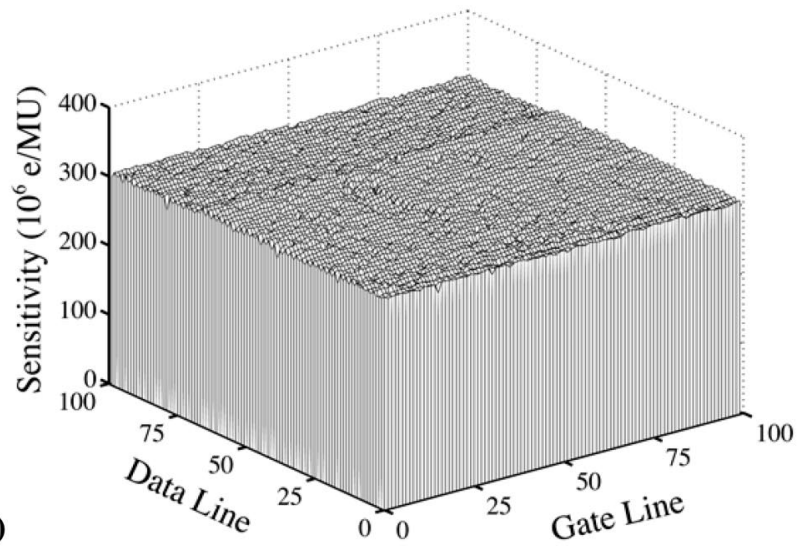

FIG. 4. (a) Signal response as a function of irradiation time (MU) for the three prototype SU- 8 detectors, measured at $6 \mathrm{MV}$. Also shown, for comparison, is previously published signal response data for Lanex Fast-B (Ref. 10) appropriately corrected for differences in measurement conditions. (b) Sensitivity profile from a $100 \times 100$ pixel region of the array underlying a central portion $(100 \times 100$ cells $)$ of the HD- 2 detector. (c) Sensitivity profile from the same $100 \times 100$ pixel region with the array coupled to a Lanex Fast-B screen.

mately equal QEs, the latter exhibits a significantly lower light output, probably due to the fact that light photons generated at the x-ray source side of the detector have to travel over a much longer distance in order to exit from the array side. On the other hand, in the case of the HD-2 configuration, the light-attenuating effect of the longer optical path is 
TABLE I. Various parameters for the SU-8 detectors, as obtained from design specifications, measurements and Monte Carlo simulations. The sensitivity values shown in the last column, were determined from the slope of the signal response of the imager (i.e., SU-8 detector+active matrix array) for each detector configuration, as shown in Fig. 4. For comparison, corresponding Lanex Fast-B values are also shown.

\begin{tabular}{lcccc}
\hline \hline Detector & $\begin{array}{c}\text { Cell height } \\
(\mu \mathrm{m})\end{array}$ & $\begin{array}{c}\text { Phosphor density } \\
\left(\mathrm{g} / \mathrm{cm}^{3}\right)\end{array}$ & $\mathrm{QE}$ & $\begin{array}{c}\text { Sensitivity } \\
\left(10^{6} \mathrm{e} / \mathrm{MU}\right)\end{array}$ \\
\hline HD-1 & 850 & 4.4 & 0.038 & 102.3 \\
HD-2 & 1900 & 4.4 & 0.069 & 92.7 \\
LD & 1900 & 2.05 & 0.039 & 44.6 \\
Lanex Fast-B & 360 & 3.67 & 0.018 & 308.9 \\
\hline \hline
\end{tabular}

largely mitigated by the higher QE (compared to LD). Nonetheless, the HD-1 configuration, despite having lower QE, shows $\sim 10 \%$ higher sensitivity than HD-2 - a result that may be explained as follows. The back surface of the SU-8 detectors is reflective, as described in Sec. III A, and serves to redirect light photons toward the array. In the case of the HD-1 detector, the back surface is closer to the array $(850 \mu \mathrm{m})$ compared to the HD-2 configuration $(1900 \mu \mathrm{m})$. As a result, the light photons reflected from the back surface of the HD-1 detector have a higher probability of escaping the detector and impinging on the array. In contrast, in the case of the HD-2 detector, light photons reflected from the back surface have to traverse a longer optical path, which significantly reduces their escape probability, thus partially negating the enhancement in signal due to higher QE.

Finally, for comparison, previously published values of the signal response of Lanex Fast-B (Ref. 10) are also shown in Fig. 4. Note that the previous measurements were performed at a SDD of $200 \mathrm{~cm}$ and have been appropriately adjusted using the inverse square correction in order to correspond to the SDD of $130 \mathrm{~cm}$ used in the present measurements. Also note that the earlier measurements were performed on a linac that used a slightly different definition of MU and therefore, the Fast-B values have been further scaled by a factor of 1.015. It can be seen that Lanex Fast-B, a screen that has been highly optimized for light output, exhibits a sensitivity $\sim 3$ times higher than that observed for the HD-1 detector.

\section{B. MTF}

Theoretically calculated and measured MTFs for the three SU-8 detector configurations are shown in Fig. 5. Figure 5(a) shows the calculated radiation MTF for each detector. It can be seen that the spread of the absorbed energy depends on the density as well as the thickness of the detector. For instance, the HD-2 detector exhibits superior radiation MTF compared to LD, which is equal in thickness $(1900 \mu \mathrm{m})$ but has approximately half the density of HD-2 - indicating that a high-density detector is more effective in limiting the lateral spread of the secondary radiation. For detectors of equal densities, the radiation MTF depends on the path-length available for the spread of the secondary radiation and, therefore, a thinner detector exhibits superior radiation MTF, as observed in the case of HD-1 compared to HD-2.
Figures 5(b)-5(d) show the measured MTFs for each detector configuration. Also shown in each figure are the pixel MTF, which is the Fourier transform of the rect function corresponding to the dimensions of the pixel photodiode $(458 \mu \mathrm{m}) ;{ }^{10}$ the theoretical maximum MTF for each detector, which is calculated as the product of the pixel MTF and the corresponding radiation MTF; and finally, for comparison, previously published Lanex Fast-B MTF data measured at $6 \mathrm{MV} .{ }^{10}$ It can be seen that the measured MTFs for the HD-1 and HD-2 detectors are lower than the respective theoretical maximum values-likely due to a small, but nonnegligible, amount of optical cross-talk between adjacent cells as well as non-ideal optical coupling between the detector and the photodiode array. For the LD detector, the measured MTF is close to the theoretical maximum, indicating that, in this case, the detector MTF is dominated by the spread of the secondary radiation and the contribution of optical effects is relatively small.

Finally, it can be seen that in each case, the SU-8 detector exhibits comparable or superior MTF relative to the Lanex Fast-B screen. These results strongly support the segmented phosphor approach by demonstrating that through the use of such a design, it is possible to significantly increase the $\mathrm{QE}$ of a phosphor-based detector and yet maintain adequate spatial resolution.

\section{NPS}

Figure 6 shows 1D noise power spectra for the SU-8 detectors, measured fluoroscopically at 1 and $2 \mathrm{MU}$ and subsequently corrected for the first-frame lag (as described in Sec. III B 2). In each case, the NPS is proportional to the amount of radiation. The NPS of the HD-2 detector exhibits a small ripple-like pattern in the higher frequency range-likely due to slight misregistration of the SU-8 cells with respect to the array pixels. Furthermore, in each case, the NPS fall-off is relatively gradual across the entire frequency range, indicating the absence of strong correlations in the data.

\section{DQE}

Figure 7 shows the DQE of the three detector configurations at 1 and $2 \mathrm{MU}$, calculated from Eq. (4), using the results of the measurements presented above. For comparison, previously measured DQE results of Lanex Fast-B at $6 \mathrm{MV}$, 


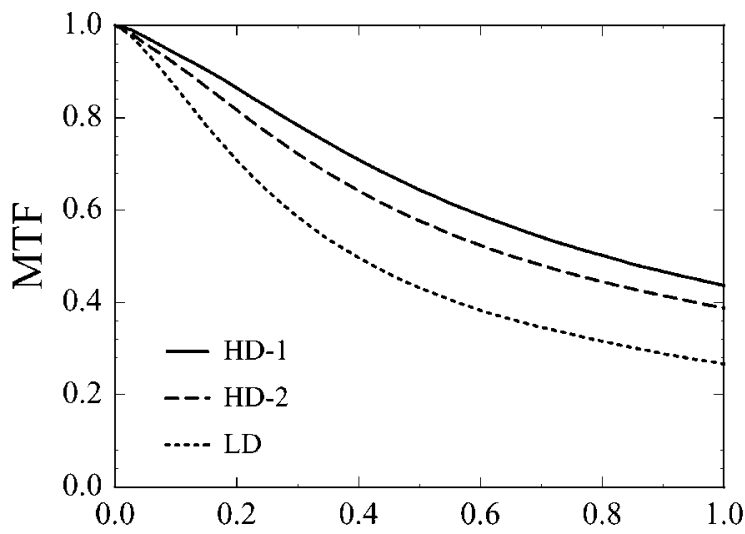

(a)

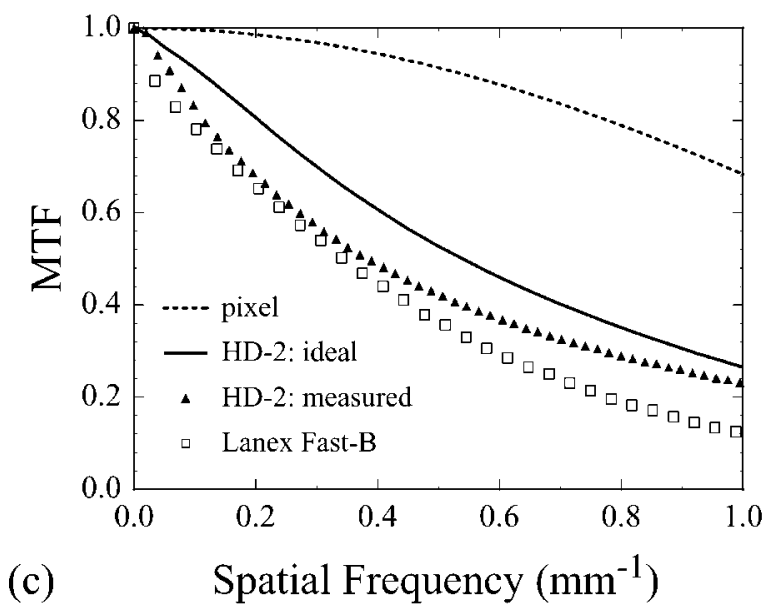

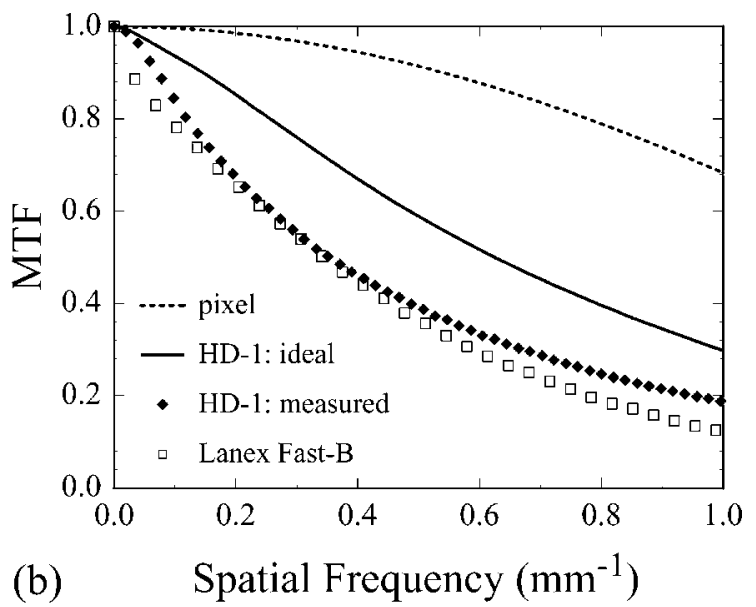

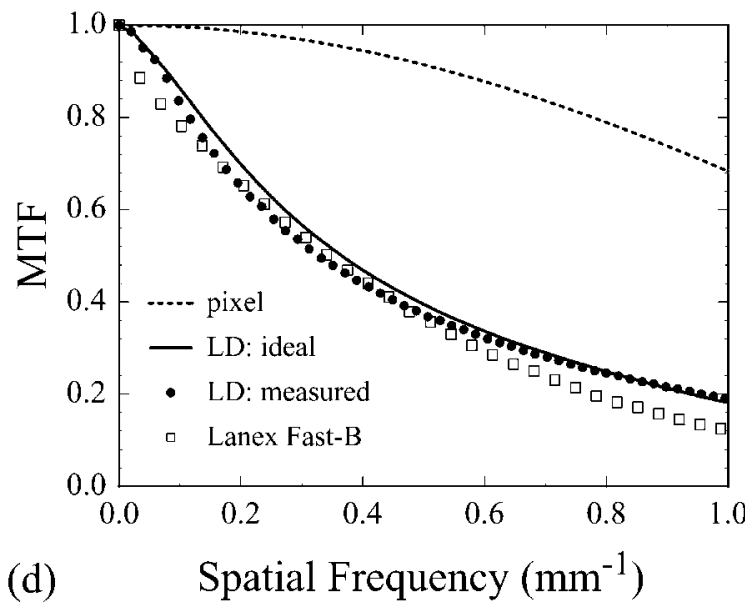

FIG. 5. Theoretical and measured modulation transfer functions. (a) Radiation MTFs for the three SU-8 detectors, obtained from Monte Carlo simulations. Measured MTFs for SU-8 detector (b) HD-1, (c) HD-2, and (d) LD. Also shown in (b), (c), and (d), are the pixel MTF and the theoretical maximum MTF, the latter of which is given by the product of the pixel MTF and the corresponding radiation MTF. The previously measured Lanex Fast-B MTF at 6 MV (Ref. 10) is also shown for comparison.

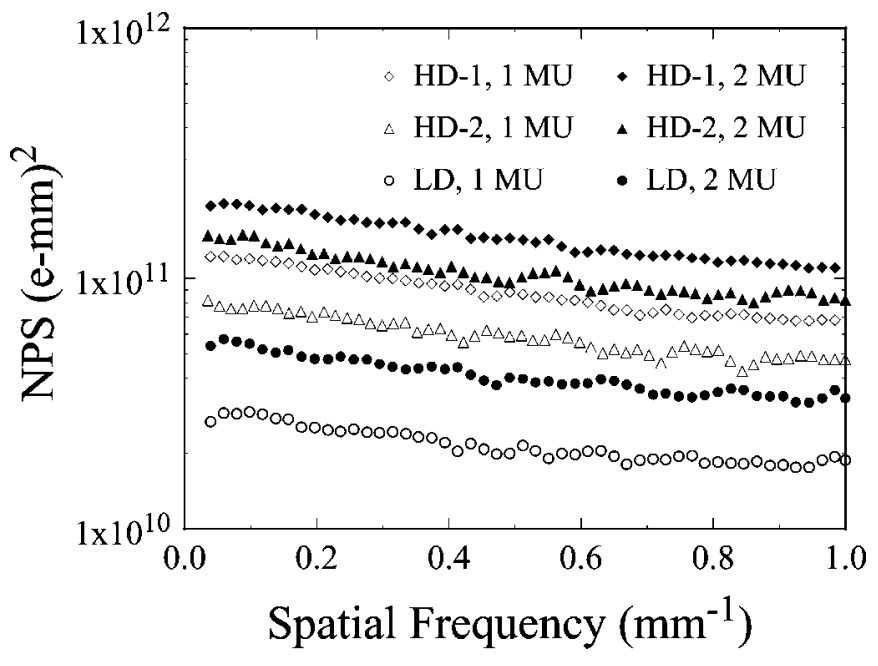

FIG. 6. Measured noise power spectra for the three SU-8 detectors, acquired at 1 and $2 \mathrm{MU}$. The measurements were performed fluoroscopically and the image data were subsequently corrected in order to account for a first-frame lag of $\sim 10 \%$.
$1 \mathrm{MU},{ }^{10}$ are also shown. It can be observed that, for the HD-2 and LD detectors, the DQE is relatively invariant with respect to increasing irradiation. At higher spatial frequencies, the HD-1 detector exhibits small relative differences $(\sim 5 \%)$ in the DQE values obtained at 1 and 2 MU. However, these differences are within the estimated experimental error of measurement under radiotherapy conditions. Thus, the DQE results for the three detectors indicate that, in each case, the imaging system is $\mathrm{x}$-ray quantum limited. The HD-2 detector, which has lower x-ray sensitivity but higher QE compared to HD-1 (see Table I), exhibits slightly superior DQE at higher spatial frequencies. In the case of the LD configuration, the relatively low $\mathrm{QE}$ results in a significant reduction of the DQE. Furthermore, the zero-frequency DQEs of all three SU-8 detectors are comparable to that of Lanex Fast-B, while at higher frequencies, the SU-8 DQEs are significantly lower. These relatively low $\mathrm{DQE}$ values can be attributed to the two noise-related mechanisms explained in Sec. II B 3-Swank noise, which reduces the DQE across all spatial frequencies and the Lubberts effect which causes the DQE to fall off at higher frequencies. 


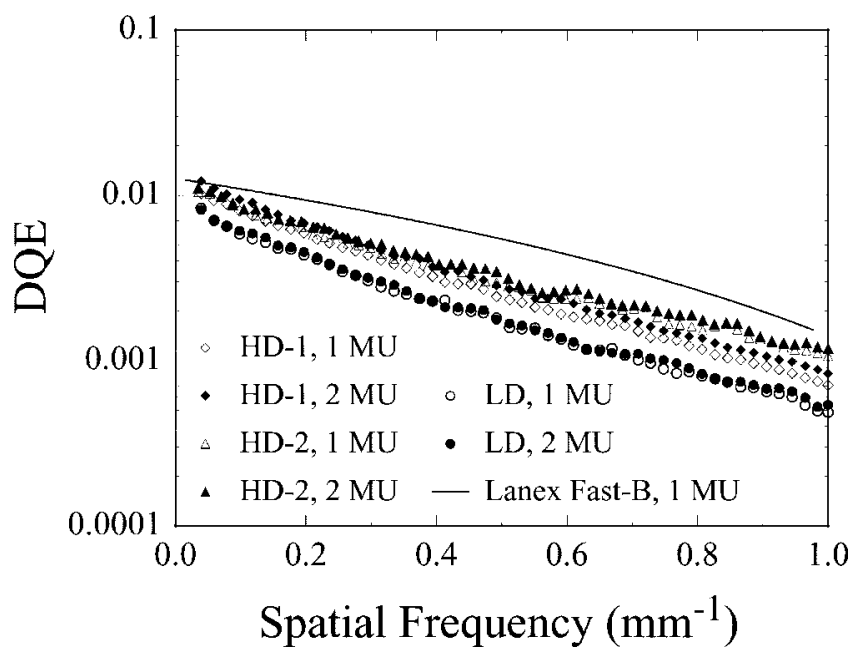

FIG. 7. DQE for the three SU-8 detectors, measured at 1 and 2 MU. Also shown are previously measured DQE results of Lanex Fast-B at $6 \mathrm{MV}$ $1 \mathrm{MU}$ (Ref. 10). The DQE of the SU-8 detectors does not change significantly with the amount of irradiation, indicating x-ray quantum limited behavior. The prototype detectors exhibit DQE values which are comparable to that of Lanex Fast-B at zero frequency, but which are significantly lower at high spatial frequencies.

The zero frequency DQE can be calculated from the product of the QE and the Swank factor. ${ }^{58}$ For instance, Lanex Fast-B which has a QE of $\sim 0.02$ (see Table I) and an estimated Swank factor of $\sim 0.5,{ }^{10}$ should yield DQE(0) of $\sim 0.01$, which is consistent with the previously measured data ${ }^{10}$ shown in Fig. 7. It follows that, in the case of the SU-8 detectors, which have up to 3 times higher QE but $\mathrm{DQE}(0)$ comparable to Fast-B, the Swank factor must presently range from 0.15 to 0.2. Such low values of Swank factor indicate very high noise levels, which can be attributed to strong depth-dependence of the optical gain, as explained in Sec. II B 3 .

The contribution of Lubberts effect towards DQE fall-off at non-zero spatial frequencies can be ascertained from the ratio $R(u)$, described in Sec. III B 4. Figure 8 shows $R(u)$, calculated using Eq. (5), for the three SU-8 detectors as well as for Lanex Fast-B. It can be seen that, at frequencies lower than $\sim 0.15 \mathrm{~mm}^{-1}$, the SU-8 detectors exhibit $R(u)$ values comparable or superior to Lanex Fast-B. However, at higher spatial frequencies, the SU-8 detectors exhibit $R(u)$ values lower than Lanex Fast-B, with the differences being more pronounced in the mid-frequency range. For instance, at $\sim 0.5 \mathrm{~mm}^{-1}, R(u)$ values for the HD-2, HD-1, and LD detectors are $\sim 0.25,0.2$, and 0.2 , respectively, compared to a value of $\sim 0.3$ for Lanex Fast-B. While such low values of $R(u)$ at high spatial frequencies cause a fall-off in the DQE (at those frequencies), the overall degradation in the DQE for SU-8 detectors (compared to Fast-B) is determined to a much larger extent by Swank noise, which reduces the DQE across the entire frequency range. As discussed above, the SU-8 detectors exhibit Swank factors that are one-half to one-third that of Lanex Fast-B. These results therefore suggest that, in the case of the SU-8 detectors, the DQE is ad-

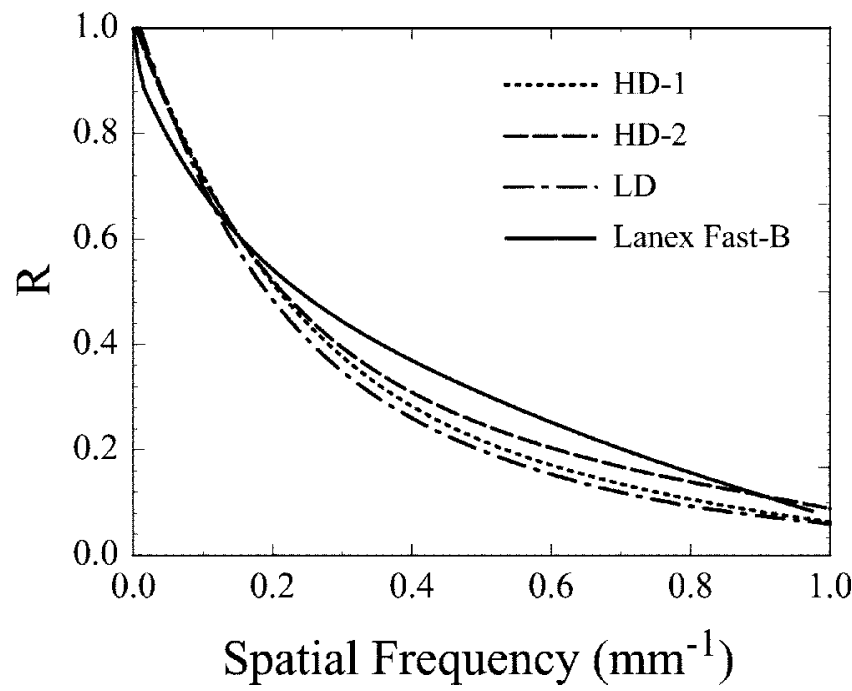

FIG. 8. Examination of the Lubberts effect in the three SU-8 detectors. The Lubberts effect causes a fall-off in the DQE at non-zero frequencies, and is quantified through the fraction $R(u)$ [defined by Eq. (5)], plotted as a function of spatial frequency. Ideally, $R(u)$ equals unity, corresponding to the absence of Lubberts effect. It can be seen that the SU- 8 detectors exhibit lower values of $R(u)$ compared to Lanex Fast-B at frequencies above $0.15 \mathrm{~mm}^{-1}$.

versely affected significantly more by Swank noise than by the Lubberts effect.

\section{DISCUSSION}

The results presented in this paper demonstrate that the segmented phosphor approach successfully overcomes the trade-off between $\mathrm{x}$-ray quantum efficiency and spatial resolution, allowing thick detectors that can achieve QEs up to at least 3 times higher than those used in current commerciallyavailable AMFPI EPIDs, while simultaneously maintaining comparable (or even, superior) spatial resolution. Consequently, such a design largely mitigates the Lubberts effect, allowing detector thicknesses to be increased up to a few $\mathrm{mm}$ - several times greater than the continuous phosphor screens that are used in current EPIDs. However, as the DQE results show, the imaging performance of these early prototypes is significantly reduced due to the presence of high levels of Swank noise. As explained in Sec. II B 3, thick phosphor-based detectors exhibit large variations in optical gain due to the strongly depth-dependent light escape efficiency. In order to reduce Swank noise, it is essential to minimize this depth dependence-a requirement that becomes more challenging with increasing detector thickness.

In principle, this goal can be accomplished by replacing the phosphor in each cell with an optically clear element of a scintillator material such as $\mathrm{CsI}(\mathrm{Tl})$ or BGO. While such strategies are currently under investigation by the authors ${ }^{59}$ as well as by others, ${ }^{20,21,29}$ practical issues related to the manufacturing complexity and cost of large-area crystalline segmented detectors make it worthwhile to examine relatively inexpensive alternatives like segmented phosphors.

One strategy to decrease the depth-dependence of the light escape efficiency in a segmented phosphor is to reduce 


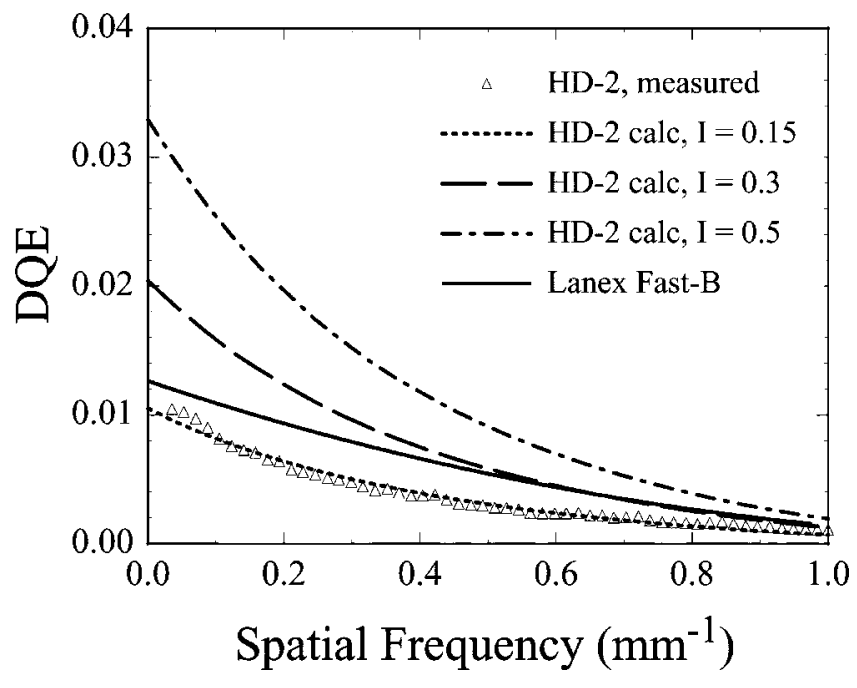

FIG. 9. Cascaded systems calculations demonstrating the performance improvement that could be achieved through reduction of Swank noise. These calculations are based on the specifications of the HD-2 detector. DQE is calculated for the present Swank noise level $(I=0.15)$ as well as lower levels of Swank noise ( $I=0.3$ and 0.5 ). These calculations indicate that for Swank noise comparable to Lanex Fast-B $(I=0.5)$, the HD-2 detector could yield $\mathrm{DQE}$ values up to 3 times higher than the presently measured values.

the amount of scatter within the phosphor grains. In a granular phosphor, light scatter occurs primarily due to multiple refraction events at the grain boundaries. Significant scatter reduction could be accomplished by dispersing the phosphor grains in an optically transparent binder material that has the same index of refraction as the phosphor. Ideally, such a configuration would enable light photons to travel along straight lines within the phosphor cell (similar to a continuous crystalline element). Another approach may be to use a combination of two phosphors that have similar QEs but significantly different light output. By carefully varying the proportion of the two phosphors as a function of cell-depth (such that the brighter phosphor has higher concentration farther away from the array side) it may be possible to compensate for the depth-dependence of the light escape efficiency. A third strategy would be to create light channels within the cells, for example, by introducing transparent glass microspheres having diameters on the order of $10 \mu \mathrm{m}$, or short glass fibers, having similar diameters, and lengths comparable to the cell height. Such light channels would serve as "high-transmission" paths for light photons generated on the x-ray side of the detector, thereby increasing the escape efficiency of these photons.

The potential improvement in DQE performance through the reduction of Swank noise, using strategies similar to those described above, may be seen in Fig. 9. The measured $\mathrm{DQE}(\mathrm{f})$ from the HD-2 detector at $1 \mathrm{MU}$ is shown, along with cascaded systems calculations for various levels of Swank noise. (The methodology of these calculations has been described in detail elsewhere. ${ }^{10,60,61}$ ) The calculations make use of the QE and the radiation MTF of the HD-2 detector, obtained through Monte Carlo simulations, as well as the empirically measured $\mathrm{x}$-ray sensitivity. The DQE curves corresponding to different levels of Swank noise were obtained by varying the gain Poisson excess parameter in the cascaded systems model, ${ }^{10,36}$ which is related to the Swank factor. For comparison, previously published DQE measurements are shown for Lanex Fast-B, which exhibits a Swank factor of $\sim 0.5$. $^{10}$

The calculations suggest that, through significant reduction of the Swank noise (to levels comparable to those of Fast-B, where the Swank noise is dominated by the XED and the AED), the HD-2 detector could achieve up to a factor of 3 higher DQE. Further improvements in DQE may be achieved by fabricating taller SU-8 cell matrices. It is anticipated that pixel-matched cell matrices up to $5 \mathrm{~mm}$ tall could be fabricated. Of course, for such high-aspect-ratio cells, maintaining low noise levels will become more challenging, perhaps necessitating the incorporation of more effective noise reduction strategies.

\section{CONCLUSION}

The segmented phosphor concept enables the realization of detectors that exhibit high QE as well as adequate spatial resolution for megavoltage imaging. In this work, detector development was conducted using a DQE-based approach. Such a methodology is well-suited for developing and evaluating novel detector designs, since it allows for the independent examination and optimization of various parameters that impact the overall imaging performance of the system. A major challenge in the further development of this design is the reduction of the presently high levels of optical Swank noise. Using innovative strategies (such as those described in the previous section), it may be possible to significantly reduce Swank noise and thereby improve the overall DQE performance of high QE segmented phosphor-based detectors.

\section{ACKNOWLEDGMENTS}

The authors would like to extend their grateful appreciation to Ian Cunningham, University of Western Ontario, for helpful suggestions on the cascaded systems model and comments regarding the manuscript. We would also like to thank John Yorkston, Eastman Kodak, Inc., for valuable discussions regarding Swank noise in granular phosphors. This work was supported by Grant No. RO1 CA 51397 from the National Institutes of Health.

\footnotetext{
a) Author to whom correspondence should be addressed. Electronic mail: asawant@umich.edu

${ }^{1}$ L. E. Antonuk et al., "Initial performance evaluation of an indirectdetection, active matrix flat-panel imager (AMFPI) prototype for megavoltage imaging," Int. J. Radiat. Oncol., Biol., Phys. 42, 437-454 (1998). ${ }^{2}$ J. J. Kruse et al., "Electronic and film portal images: A comparison of landmark visibility and review accuracy," Int. J. Radiat. Oncol., Biol., Phys. 54, 584-591 (2002).

${ }^{3}$ L. E. Antonuk, "Electronic portal imaging devices: A review and historical perspective of contemporary technologies and research," Phys. Med. Biol. 47, R31-R65 (2002).

${ }^{4}$ T. Falco, H. Wang, and B. G. Fallone, "Preliminary study of a metal/aSe-based portal detector," Med. Phys. 25, 814-823 (1998).

${ }^{5}$ M. Lachaine and B. G. Fallone, "Monte Carlo detective quantum efficiency and scatter studies of a metal/a-Se portal detector," Med. Phys. 25, 1186-1194 (1998).
} 
${ }^{6}$ M. Lachaine, E. Fourkal, and B. G. Fallone, "Detective quantum efficiency of a direct-detection active matrix flat panel imager at megavoltage energies," Med. Phys. 28, 1364-1372 (2001).

${ }^{7}$ G. Pang, D. L. Lee, and J. A. Rowlands, "Investigation of a direct conversion flat panel imager for portal imaging," Med. Phys. 28, 2121-2128 (2001).

${ }^{8}$ G. Pang and J. A. Rowlands, "Development of high quantum efficiency, flat panel, thick detectors for megavoltage x-ray imaging: A novel directconversion design and its feasibility," Med. Phys. 31, 3004-3016 (2004).

${ }^{9}$ P. Munro and D. C. Bouius, "X-ray quantum limited portal imaging using amorphous silicon flat-panel arrays," Med. Phys. 25, 689-702 (1998).

${ }^{10}$ Y. El-Mohri, K.-W. Jee, L. E. Antonuk, M. Maolinbay, and Q. Zhao, "Determination of the detective quantum efficiency of a prototype, megavoltage indirect detection, active matrix flat-panel imager," Med. Phys 28, 2538-2550 (2001).

${ }^{11}$ B. Wowk, T. Radcliffe, K. W. Leszczynski, S. Shalev, and R. Rajapakshe, "Optimization of metal/phosphor screens for online portal imaging," Med. Phys. 21, 227-235 (1994).

${ }^{12}$ B. Wowk and S. Shalev, "Thick phosphor screens for on-line portal imaging,"Med. Phys. 21, 1269-1276 (1994).

${ }^{13}$ J.-P. Bissonnette, I. A. Cunningham, and P. Munro, "Optimal phosphor thickness for portal imaging," Med. Phys. 24, 803-814 (1997).

${ }^{14} \mathrm{~F}$. Cremers et al., "Performance of electronic portal imaging devices (EPIDs) used in radiotherapy: Image quality and dose measurements," Med. Phys. 31, 985-996 (2004).

${ }^{15}$ E. Samei and M. J. Flynn, "An experimental comparison of detector performance for direct and indirect digital radiography systems," Med. Phys. 30, 608-622 (2003).

${ }^{16}$ E. Samei, M. J. Flynn, H. G. Chotas, and J. T. Dobbins, "DQE of direct and indirect digital radiographic systems," Proc. SPIE 4320, 189-197 (2001).

${ }^{17}$ P. R. Granfors and R. Aufrichtig, "Performance of a $41 \times 41-\mathrm{cm}^{2}$ amorphous silicon flat panel $\mathrm{x}$-ray detector for radiographic imaging applications," Med. Phys. 27, 1324-1331 (2000).

${ }^{18}$ D. L. Lee et al., "Filtered gain calibration and its effect on frequencydependent DQE and image quality in Se-based general radiography and full-field mammographic digital imaging," Proc. SPIE 4320, 121-126 (2001).

${ }^{19}$ B. Wowk, S. Shalev, and T. Radcliffe, "Grooved phosphor screens for online portal imaging," Med. Phys. 20, 1641-1651 (1993).

${ }^{20}$ M. A. Mosleh-Shirazi et al., "Rapid portal imaging with a high-efficiency, large field-of-view detector," Med. Phys. 25, 2333-2346 (1998).

${ }^{21}$ M. A. Mosleh-Shirazi, W. Swindell, and P. M. Evans, "Optimization of the scintillation detector in a combined 3D megavoltage CT scanner and portal imager," Med. Phys. 25, 1880-1890 (1998).

${ }^{22}$ A. Sawant et al., "Theoretical analysis and experimental evaluation of a CsI(Tl) based electronic portal imaging system," Med. Phys. 29, 10421053 (2002).

${ }^{23}$ S. Samant, W. Zheng, F. DiBianca, H. Zeman, and J. Laughter, "A new calibration technique for KCD-based megavoltage imaging," Proc. SPIE 3659, 779-792 (1999).

${ }^{24}$ F. DiBianca, S. Samant, J. Laughter, J. Rasmussen, and C. Rodriguez, "Use of a kinestatic charge detector for megavoltage portal imaging," Proc. SPIE 3032, 195-201 (1997).

${ }^{25} \mathrm{~A}$. Brahme et al., "Evaluation of a GEM and CAT-based detector for radiation therapy beam monitoring," Nucl. Instrum. Methods Phys. Res. A 454, 136-141 (2000).

${ }^{26} \mathrm{~J}$. Ostling et al., "Novel detector for portal imaging in radiation therapy," Proc. SPIE 3977, 84-95 (2000).

${ }^{27} \mathrm{C}$. Iacobaeus et al., "A novel portal imaging device for advanced radiation therapy," IEEE Trans. Nucl. Sci. 48, 1496-1502 (2001).

${ }^{28}$ T. T. Monajemi, S. Steciw, B. G. Fallone, and S. Rathee, "Modeling scintillator-photodiodes as detectors for megavoltage CT," Med. Phys. 31, 1225-1234 (2004).

${ }^{29}$ E. J. Seppi et al., "Megavoltage cone-beam computed tomography using a high-efficiency image receptor," Int. J. Radiat. Oncol., Biol., Phys. 55, 793-803 (2003).

${ }^{30}$ G. Pang and J. A. Rowlands, "Development of high quantum efficiency flat panel detectors for portal imaging: Intrinsic spatial resolution," Med. Phys. 29, 2274-2285 (2002).

${ }^{31}$ A. L. Boyer et al., "A review of electronic portal imaging devices (EPIDs)," Med. Phys. 19, 1-16 (1992).

${ }^{32}$ P. Munro, "Portal imaging technology: Past, present, and future," Semin
Radiat. Oncol. 5, 115-133 (1995).

${ }^{33}$ J. Beutel, H. L. Kundel, and R. L. Van Metter, Handbook of Medical Imaging (SPIE, Washington, 2000).

${ }^{34}$ I. Cunningham, "Applied Linear-Systems Theory," in Handbook of Medical Imaging, edited by J. Beutel, H. L. Kundel, and R. L. Van Metter (SPIE, Washington, 2000), pp. 79-159.

${ }^{35}$ M. Rabbani, R. Shaw, and R. Van Metter, "Detective quantum efficiency of imaging systems with amplifying and scattering mechanisms," J. Opt. Soc. Am. A 4, 895-901 (1987).

${ }^{36}$ I. A. Cunningham, M. S. Westmore, and A. Fenster, "A spatial-frequency dependent quantum accounting diagram and detective quantum efficiency model of signal and noise propagation in cascaded imaging systems," Med. Phys. 21, 417-427 (1994).

${ }^{37}$ R. K. Swank, "Absorption and noise in x-ray phosphors," J. Appl. Phys. 44, 4199-4203 (1973).

${ }^{38}$ M. Drangova and J. A. Rowlands, "Optical factors affecting the detective quantum efficiency of radiographic screens," Med. Phys. 13, 150-157 (1986).

${ }^{39}$ G. Lubberts, "Random noise produced by x-ray fluorescent screens," J. Opt. Soc. Am. 58, 1475-1483 (1968).

${ }^{40}$ R. M. Nishikawa, M. J. Yaffe, and R. B. Holmes, "Effect of finite phosphor thickness on detective quantum efficiency," Med. Phys. 16, 773780 (1989).

${ }^{41} \mathrm{~K}$. Y. Lee et al., "Micromachining applications of a high resolution ultrathick photoresist," J. Vac. Sci. Technol. B 13, 3012-3016 (1995).

${ }^{42}$ J. H. Daniel et al., "Large area MEMS fabrication with thick SU-8 photoresist applied to an x-ray image sensor array," Proc. SPIE 4174, 40-48 (2000).

${ }^{43}$ J. H. Daniel et al., "Microelectromechanical system fabrication technology applied to large area X-ray image sensor arrays," J. Vac. Sci. Technol. A 19, 1219-1223 (2001).

${ }^{44}$ J. H. Daniel et al., "MEMS materials and fabrication technology for large areas: The example of an X-ray imager," Mater. Res. Soc. Symp. Proc. 657, EE9.3.1-EE9.3.6 (2001).

${ }^{45} \mathrm{~W}$. Huang et al., "An asynchronous, pipelined, electronic acquisition system for active matrix flat-panel imagers (AMFPIs)," Nucl. Instrum. Methods Phys. Res. A 431, 273-284 (1999).

${ }^{46} \mathrm{M}$. Maolinbay et al., "Additive noise properties of active matrix flat-panel imagers,” Med. Phys. 27, 1841-1854 (2000).

${ }^{47} \mathrm{H}$. Fujita et al., "A simple method for determining the modulation transfer-function in digital radiography," IEEE Trans. Med. Imaging 11, 34-39 (1992).

${ }^{48}$ L. E. Antonuk et al., "Demonstration of megavoltage and diagnostic x-ray imaging with hydrogenated amorphous silicon arrays," Med. Phys. 19, 1455-1466 (1992).

${ }^{49}$ J. H. Siewerdsen et al., "Signal, noise power spectrum, and detective quantum efficiency of indirect-detection flat-panel imagers for diagnostic radiology," Med. Phys. 25, 614-628 (1998).

${ }^{50} \mathrm{P}$. R. Granfors et al., "Performance of a $41 \times 41 \mathrm{~cm}^{2}$ amorphous silicon flat panel $\mathrm{x}$-ray detector designed for angiographic and $\mathrm{R} \& \mathrm{~F}$ imaging applications," Med. Phys. 30, 2715-2726 (2003).

${ }^{51}$ P. R. Granfors and R. Aufrichtig, "DQE(f) of an amorphous-silicon flatpanel x-ray detector: Detector parameter influences and measurement methodology," Proc. SPIE 3977, 2-13 (2000).

${ }^{52}$ W. R. Nelson, and D. W. O. Rogers, "Structure and operation of the EGS4 code system," in Monte Carlo Transport of Electrons and Photons Below $50 \mathrm{MeV}$, edited by T. M. Jenkins, W. R. Nelson, A. Rindi, A. E. Nahum, and D. W. O. Rogers (Plenum, New York, 1989), pp. 287-306.

${ }^{53}$ D. A. Jaffray, J. J. Battista, A. Fenster, and P. Munro, "Monte Carlo studies of $\mathrm{x}$-ray-energy absorption and quantum-noise in megavoltage transmission radiography," Med. Phys. 22, 1077-1088 (1995).

${ }^{54} \mathrm{D}$. Sheikh-Bagheri, "Monte Carlo study of photon beams from medical linear accelerators: Optimization, benchmark, and spectra," Ph.D. thesis, Department of Physics, Carleton University, 1999.

${ }^{55}$ I. Kawrakow and D. W. O. Rogers, "The EGSnrc Code System: Monte Carlo simulation of electron and photon transport," Technical Report No. PIRS-701, National Research Council of Canada, Ottawa, Canada, 2000.

${ }^{56}$ J. A. Treurniet, B. R. B. Walters, and D. W. O. Rogers, "BEAMnrc, DOSXYZnrc and BEAMDP GUI User's Manual," NRC Report No. PIRS 0623(rev B), 2001.

${ }^{57}$ L. E. Antonuk et al., "Light-response characteristics of amorphous silicon arrays for megavoltage and diagnostic imaging," Mater. Res. Soc. Symp. Proc. 219, 531-536 (1991). 
${ }^{58}$ J. Rowlands and J. Yorkston, "Flat panel detectors for digital radiography," in Handbook of Medical Imaging, edited by J. Beutel, H. L. Kundel, and R. L. Van Metter (SPIE, Washington, 2000), pp. 328.

${ }^{59}$ A. R. Sawant et al., "Exploring new frontiers in x-ray quantum limited portal imaging using active matrix flat-panel imagers (AMFPIs)," Proc. SPIE 5030, 478-489 (2003).
${ }^{60}$ L. E. Antonuk et al., "Strategies to improve the signal and noise performance of active matrix, flat-panel imagers for diagnostic x-ray applications," Med. Phys. 27, 289-306 (2000).

${ }^{61}$ J. H. Siewerdsen et al., "Empirical and theoretical investigation of the noise performance of indirect detection, active matrix flat-panel imagers (AMFPIs) for diagnostic radiology," Med. Phys. 24, 71-89 (1997). 